\title{
Lessons Learned from a Fulminant Case of Reversible Cerebral Vasoconstriction Syndrome: Past Medical History Misleads the Diagnosis and Intra-Arterial Milrinone Offers Diagnostic Utility
}

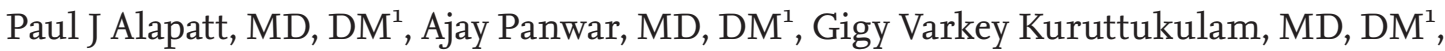 \\ Kaushik Sundar, MD, DM ${ }^{2}$ \\ ${ }^{1}$ Department of Neurology, Rajagiri Hospital, Kochi, India \\ ${ }^{2}$ Department of Neurology, Rabindranath International Institute of Cardiac Sciences, Kolkata, India
}

\begin{abstract}
A 34-year-old post-partum female having dermatomyositis developed headache and became comatose after a seizure episode. Magnetic resonance imaging of brain showed a massive left ganglio-capsular bleed for which decompressive surgery was done. Computed tomographic angiography showed multiple foci of narrowing and irregularities in distal cerebral vessels. In view of dermatomyositis, the diagnosis of vasculitis was considered and pulse therapy of intravenous methylprednisolone was started. The patient, however, showed no improvement and developed new brain infarcts. She was subsequently taken up for a diagnostic cerebral angiography which showed multifocal severe narrowing in bilateral major cerebral arteries. These angiographic abnormalities showed excellent reversibility to intra-arterial milrinone and hence, reversible cerebral vasoconstriction syndrome (RCVS) was diagnosed. Normal angiographic findings in the first week do not rule out the disease and a repeat angiography should be considered if the clinical suspicion of the RCVS is high. Intra-arterial milrinone has a high diagnostic utility.
\end{abstract}

Key Words: Reversible cerebral vasoconstriction syndrome; Milrinone; Diagnostic cerebral angiography; Vasculitis

\section{INTRODUCTION}

The diagnosis of reversible cerebral vasoconstriction syndrome (RCVS) often may be misleading and delayed because of the dynamic evolution of clinico-radiological features. RCVS is known to have an initial week of a hemorrhagic phase, which is later followed by ischemic complications. The angiographic features of RCVS are often best demon- strable only in the ischemic phase. Here, we present an unusual case of large basal ganglia hemorrhage in which the diagnosis was initially considered vasculitis. The diagnosis of RCVS was subsequently established by excellent angiographic reversibility to intra-arterial milrinone.

\section{Correspondence to: Ajay Panwar, MD, DM Department of Neurology, Rajagiri Hospital, Chunangamvely, Aluva, Kochi 683112, Kerala, India Tel: +91-8948800337 Fax: +91-40-27154758 \\ E-mail: ajay19panwar@gmail.com}

Received: November 18, 2020 Revised: December 12, 2020 Accepted: December 14, 2020

Copyright $\odot 2021$ Korean Society of Interventional Neuroradiology This is an Open Access article distributed under the terms of the Creative Commons Attribution Non-Commercial License (http://creativecommons.org/licenses/by-nc/4.0) which permits unrestricted non-commercial use, distribution, and reproduction in any medium, provided the original work is properly cited.

pISSN 2093-9043 eISSN 2233-6273 


\section{CASE REPORT}

A 34-year-old pregnant female (gravida 4, para 2, abortion 1 , intrauterine death 1) at 32 weeks of gestation presented with preterm premature rupture of membranes. The patient had a background history of dermatomyositis with interstitial lung disease in remission for the last 4 years. She was initially optimized on steroids and mycophenolate mofetil and subsequently switched to tacrolimus after detection of pregnancy. She underwent an uneventful emergency cesarean section under regional anesthesia. During the next day, she developed a moderately severe headache, which was holocranial and continuous. She had no features of raised intracranial tension. Her headache was not relieved with analgesics, and on the second postoperative day, she developed a generalized seizure during sleep. She continued to be in a comatose state following the seizure with a Glasgow Coma Scale score of eye opening (E)1, verbal response $(V) 2$, best motor response $(M) 5$. Serial blood pressure readings were normal in the post-partum period. Examination revealed bilateral pupils $1.5 \mathrm{~mm}$ in size and a sluggish reaction to light, along with a paucity of movements in the right extremities. Magnetic resonance imaging of the brain showed a large intra-parenchymal hematoma involving the left ganglio-capsular and frontotemporal regions causing a midline shift of $16 \mathrm{~mm}$ with intra-ventricular extension and uncal herniation (Fig. 1A). She underwent emergency craniotomy and hematoma evacuation. A postoperative computed tomography (CT) scan of the brain showed good evacuation of the hematoma and correction of midline shift
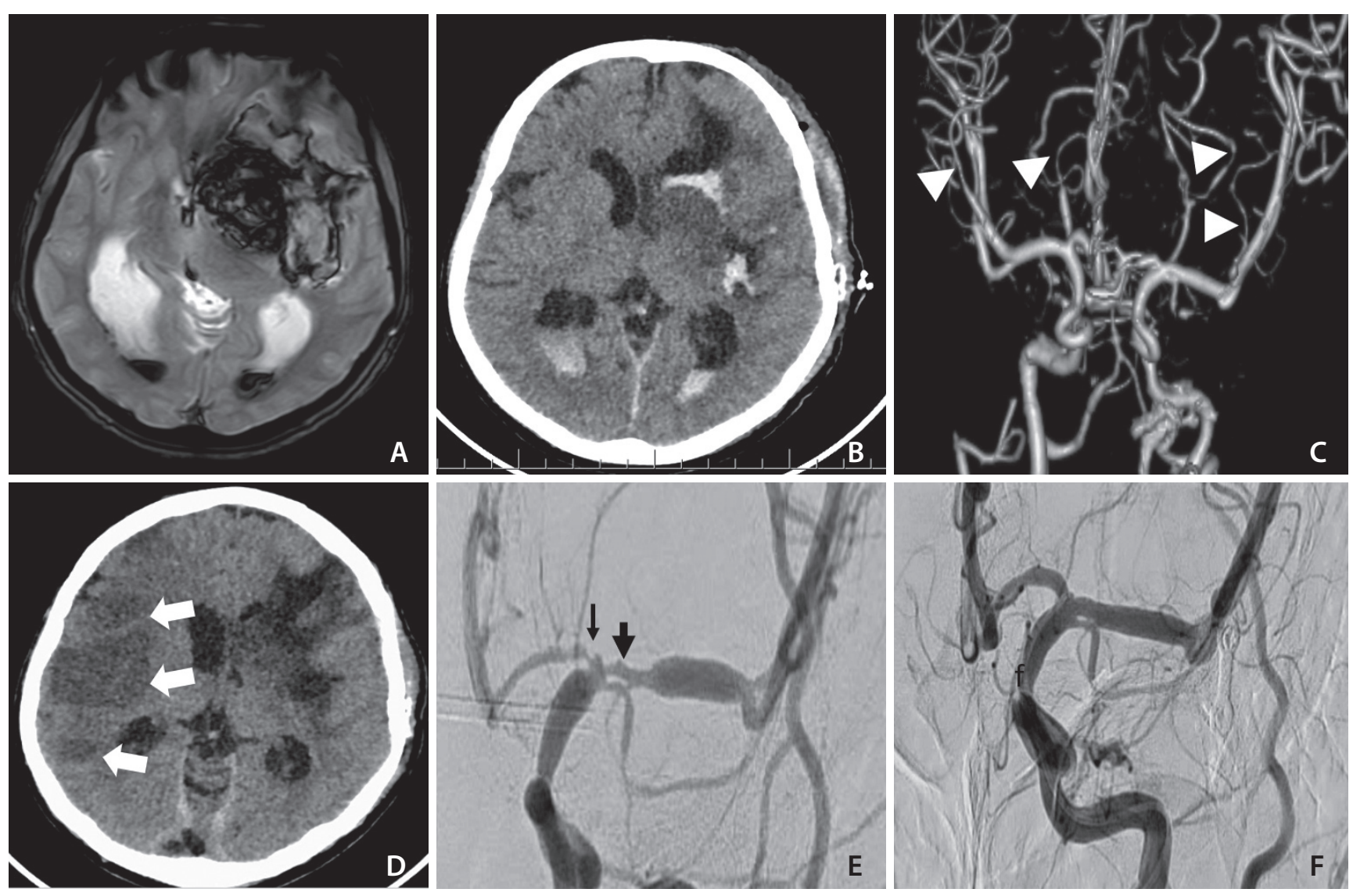

Fig. 1. (A) Magnetic resonance imaging of brain showing large intra-parenchymal hematoma involving left ganglio-capsular and frontotemporal regions with significant midline shift. (B) Post-operative computed tomography (CT) scan of brain showing hematoma evacuation. (C) CT angiography demonstrating multifocal luminal irregularities and narrowing (white arrowheads) of distal branches of bilateral middle and posterior cerebral arteries. (D) CT brain on twelfth postoperative day showing multiple acute infarcts (white arrows) in right middle cerebral arteries (MCA) territory. (E) Digital subtraction angiography performed in the second week showing severe proximal narrowing of left MCA (black thick arrow) and the anterior cerebral arteries (ACA) (black thin arrow). (F) Post intra-arterial milrinone injection of left internal carotid artery showing excellent reversibility of MCA and ACA abnormalities. 
(Fig. 1B). Computed tomography angiography (CTA) showed mild focal narrowing and irregularities in distal branches of bilateral middle cerebral arteries, anterior cerebral arteries, and posterior cerebral arteries (Fig. 1C). In the background of dermatomyositis and radiological findings, the possibility of vasculitis was considered. Erythrocyte sedimentation rate was $25 \mathrm{~mm} / \mathrm{h}$ and C-reactive protein was $6 \mathrm{mg} / \mathrm{L}$. Autoimmune workup for vasculitis including antinuclear antibody, antineutrophil cytoplasmic antibodies, rheumatoid factor, and antiphospholipid antibodies were negative. Viral serology for hepatitis B, hepatitis C, and human immunodeficiency virus was negative. Intravenous pulse methylprednisolone was given for 3 days; however, she continued to be in a deep coma. Serial follow-up CT scans of the brain did not show any hydrocephalus or bleed. Electroencephalogram did not show any epileptiform abnormalities. On the twelfth postoperative day, an examination revealed the patient to be in a quadriparetic state because of a newly developed left-sided weakness. A consequent $\mathrm{CT}$ brain showed multiple infarcts in the territories of the right anterior and middle cerebral arteries (Fig. 1D). Digital subtraction angiography (DSA) done on the same day showed severe narrowing and irregularity of bilateral anterior, middle, and posterior cerebral arteries, which showed excellent reversibility to intra-arterial milrinone infusion over 15 minutes (Fig. 1E, F). She was continued on intravenous milrinone infusion for 7 days. The diagnosis of RCVS was made secondary to her post-partum status, along with a possible etiological implication of tacrolimus therapy. Despite an $\mathrm{RCVS}_{2}$ score of only 4, which implies a low diagnostic sensitivity for the syndrome, the diagnosis of RCVS as opposed to vasculitis, was supported by excellent reversibility of angiographic abnormalities to milrinone. 'Subsequently, the patient gradually recovered from her comatose state and started moving her right upper and lower limbs. CTA done before the discharge from the hospital in the sixth postoperative week showed no significant abnormalities. She was conscious and alert at the time of discharge with a power of grade $3 / 5$ and 2/5 in her right and left extremities, respectively. Her neurological recovery is expected to be delayed due to bihemispheric dysfunction and multiple infarcts in the right cerebral hemisphere before milrinone therapy.

\section{DISCUSSION}

RCVS is a clinical and radiological syndrome characterized by sudden severe headache and reversible multifocal narrowing of cerebral arteries. ${ }^{2}$ This syndrome is often considered to have a benign outcome; however, major strokes resulting in severe disability and death have been reported. ${ }^{3}$ A recent systematic review has observed intracerebral hemorrhage $(\mathrm{ICH})$ as the most common presentation (42\%) in fatal RCVS cases, followed by encephalopathy and/or headaches in $25 \%$, and ischemic stroke and posterior reversible encephalopathy syndrome in $16.5 \%$ each. ${ }^{4}$ The same review noted that half of these fatal cases initially presented with a nonspecific headache (NSH). Our patient also had NSH as the initial manifestation. Diagnosis of RCVS can often be delayed due to the dynamic natural history and radiological features of the disease. Stroke may occur after a few days of the initial normal brain imaging. Further, angiographic vasoconstriction may show up the best only after 2-3 weeks of the clinical onset. $^{3}$

In the present case, we were deceived initially by the mild distal focal narrowings on CTA, which steered our diagnosis towards cerebral vasculitis especially against the backdrop of dermatomyositis. Central nervous system vasculitis is known to be associated with $\mathrm{ICH}^{5}$ Consequently, we started pulse therapy of intravenous methylprednisolone; the patient, however, continued to be in a comatose state. She instead developed new cerebral infarcts, and hence underwent DSA, which revealed severe multi-focal narrowings that demonstrated a striking reversal on the administration of intra-arterial milrinone. So, a probable diagnosis of RCVS was established, which was further supported by the normal CTA in the sixth postoperative week. In the retrospect, however, we understand that corticosteroid use may result in a poor outcome in RCVS and should be avoided, although, in our case, it was used considering the possibility of vasculitis before the diagnosis got confirmed. ${ }^{6,7}$

This case of fulminant RCVS was unusual and left us with several learning lessons. First, the diagnosis was delayed because of the characteristically dynamic natural history of the disease. Clinically, our patient presented with a non-specific headache initially, as against a thunderclap headache which is classically seen in RCVS. Furthermore, this disease is described as having an initial hemorrhagic phase followed by an ischemic phase in the second week..$^{8-10}$ It is also characterized by minimal angiographic findings in the initial hemorrhagic phase when the vasospasm is in the distal vessels, which is followed by proximal migration of vasospasm causing ischemic strokes in the second phase. ${ }^{8}$ Although 
the evolution of radiological changes with time has been described in the literature, this case highlights how this may cause confusion in the diagnosis and require caution in evaluating such cases. Normal angiography in the first week may not rule out the disease and a repeat angiography may be warranted if the clinical suspicion of the RCVS is high. Further, our patient had a massive ganglio-capsular regional bleed, which has rarely been mentioned in the medical literature. RCVS commonly presents in the hemorrhagic phase as $\mathrm{SAH}$ and cortical bleeds. ${ }^{3}$ It may be noted that the presence of a large basal ganglia hemorrhage should not preclude one from considering RCVS as a diagnosis, especially in an appropriate clinical setting such as a post-partum state. The exposure of our patient to tacrolimus further strengthened the associated risk.

Lastly, the high diagnostic utility of intra-arterial milrinone for RCVS has a mere anecdotal mention in the literature." We, however, observed it to have a dramatic role in reversing the vasoconstriction; and thus, it has a remarkable potential for differentiating RCVS from vasculitis. In the medical literature though, angiographic response to intra-arterial vasodilators is varied and there is a lack of strong evidence for the therapeutic role of the same in RCVS. ${ }^{11-14}$ Randomized studies conducted on the role of Nimodipine for the same have not shown any significant benefit. 15,16 Thus, our case may be considered as a potential source for conducting further randomized studies in this regard. It would be worthwhile to see if the diagnostic utility of milrinone as observed in our case can be established further with well-designed prospective studies. In conclusion, intra-arterial milrinone appears to have a significant diagnostic utility for RCVS.

\section{Fund}

None.

\section{Ethics Statement}

This study was a case report, ethical approval was not required; however, informed consent was taken from the patient's close relatives for presenting the patient's data as a report.

\section{Conflicts of Interest}

The authors have no conflicts to disclose.

\section{Author Contributions}

Concept and design: PJA, AP, GVK, and KS. Analysis and in- terpretation: PJA, AP, and GVK. Data collection: PJA and AP. Writing the article: PJA, AP, and KS. Critical revision of the article: PJA, AP, GVK, and KS. Final approval of the article: PJA, AP, GVK, and KS. Overall responsibility: AP.

\section{ORCID}

Paul J Alapatt: https://orcid.org/0000-0002-2051-5664

Ajay Panwar: https://orcid.org/0000-0002-8938-9168

Gigy Varkey Kuruttukulam: https://orcid.org/0000-0003-1614-5434

Kaushik Sundar: https://orcid.org/0000-0003-0108-1810

\section{REFERENCES}

1. Rocha EA, Topcuoglu MA, Silva GS, Singhal AB. RCVS2 score and diagnostic approach for reversible cerebral vasoconstriction syndrome. Neurology 2019;92:e639-e647

2. Saini M, Jeerakathil T, Butcher K. Reversible cerebral vasoconstriction syndrome. Neurol India 2009;57:63-65

3. Ducros A. Reversible cerebral vasoconstriction syndrome. Lancet Neurol 2012;11:906-917

4. Valencia-Mendoza M, Ramírez-Rodríguez N, Vargas-Avila N, Peña-Ortiz A, Corzo-Villamizar M, Serna-Ramírez L, et al. Fatal reversible cerebral vasoconstriction syndrome: a systematic review of case series and case reports. J Clin Neurosci 2019;70:183188

5. Salvarani C, Brown RD Jr, Calamia KT, Christianson TJ, Huston J 3rd, Meschia JF, et al. Primary central nervous system vasculitis presenting with intracranial hemorrhage. Arthritis Rheum 2011;63:3598-3606

6. Singhal AB, Hajj-Ali RA, Topcuoglu MA, Fok J, Bena J, Yang D, et al. Reversible cerebral vasoconstriction syndromes: analysis of 139 cases. Arch Neurol 2011;68:1005-1012

7. Singhal AB, Topcuoglu MA. Glucocorticoid-associated worsening in reversible cerebral vasoconstriction syndrome. Neurology 2017;88:228-236

8. Xing B, Lenck S, Krings T, Hengwei J, Jaigobin CS, Schaafsma JD. Angiographic characteristics of hemorrhagic and ischemic phases of reversible cerebral vasoconstriction syndrome. Clin Neuroradiol 2020;30:85-89

9. Ducros A, Fiedler U, Porcher R, Boukobza M, Stapf C, Bousser MG. Hemorrhagic manifestations of reversible cerebral vasoconstriction syndrome: frequency, features, and risk factors. Stroke 2010;41:2505-2511

10. Mrozek $S$, Lonjaret $L$, Jaffre $A$, Januel AC, Raposo N, Boetto $S$, et al. Reversible cerebral vasoconstriction syndrome with intra- 
cranial hypertension: should decompressive craniectomy be considered? Case Rep Neurol 2017;9:6-11

11. Bouchard M, Verreault S, Gariépy JL, Dupré N. Intra-arterial milrinone for reversible cerebral vasoconstriction syndrome. Headache 2009;49:142-145

12. Nowak DA, Rodiek SO, Henneken S, Zinner J, Schreiner R, Fuchs $\mathrm{HH}$, et al. Reversible segmental cerebral vasoconstriction (Call-Fleming syndrome): are calcium channel inhibitors a potential treatment option? Cephalalgia 2003;23:218-222

13. Mijalski C, Dakay K, Miller-Patterson C, Saad A, Silver B, Khan M. Magnesium for treatment of reversible cerebral vasoconstriction syndrome: case series. Neurohospitalist 2016;6:111-113
14. Linn J, Fesl G, Ottomeyer C, Straube A, Dichgans M, Bruckmann $\mathrm{H}$, et al. Intra-arterial application of nimodipine in reversible cerebral vasoconstriction syndrome: a diagnostic tool in select cases? Cephalalgia 2011;31:1074-1081

15. Ducros A, Boukobza M, Porcher R, Sarov M, Valade D, Bousser MG. The clinical and radiological spectrum of reversible cerebral vasoconstriction syndrome. A prospective series of 67 patients. Brain 2007;130(Pt 12):3091-3101

16. Chen SP, Fuh JL, Chang FC, Lirng JF, Shia BC, Wang SJ. Transcranial color doppler study for reversible cerebral vasoconstriction syndromes. Ann Neurol 2008;63:751-757 